\title{
High temperature performance of an optical microfibre coupler and its potential use as a sensor
}

\author{
Pengfei Wang ${ }^{1,2},{ }^{*}$ Ming Ding ${ }^{1}$, Gilberto Brambilla ${ }^{1}$, Yuliya Semenova ${ }^{2}$, Qiang $\mathrm{Wu}^{2}$, \\ Gerald Farrell $^{2}$ \\ ${ }^{1}$ Optoelectronics Research Centre, University of Southampton, Southampton SO17 1BJ, \\ United Kingdom \\ ${ }^{2}$ Photonic Research Centre, Dublin Institute of Technology, Kevin Street, Dublin 8, \\ Ireland \\ *Corresponding author: pw3y09@orc.soton.ac.uk
}

\begin{abstract}
The dependence of the transmission spectrum of an optical microfibre coupler at high temperatures is investigated experimentally. Results show a temperature sensitivity up to $36.59 \mathrm{pm} /{ }^{\circ} \mathrm{C}$ at a taper waist outer diameter of $2.5 \mu \mathrm{m}$; such a temperature-dependent performance can be utilized for temperature sensing up to $1000^{\circ} \mathrm{C}$.
\end{abstract}

Keywords: Fibre optics, microfibre, fibre coupler, temperature dependence

In recent years, optical microfibres have attracted tremendous interest for a range of both filtering and sensing applications due to the enormous progress that has occurred in the fabrication of low-loss optical micro/nano wires, which allows for efficient optical beam confinement and low-loss evanescent waveguiding [1]. Optical micro-devices fabricated from sub-wavelength fibres offer several potential benefits by comparison to conventional integrated micro-photonic devices, such as low insertion loss, inherent fibre compatibility, ease of integration, stability and flexibility. These optical properties have been shown to be advantageous for a wide range of applications including high-sensitivity optical sensors, nonlinear optics, atom trapping, micro/nano-scale photonic devices and for evanescent coupling to planar waveguides or microcavities [1].

In a recent work [2], the authors have presented a broadband bi-conical $2 \times 2$ optical microfibre coupler made from conventional telecom singlemode fibres that effectively suppresses any higher-order mode present at the input fibre and provides efficient power splitting into the fundamental mode at the two output ports. The 
microfibre coupler has many potential applications, for example in high performance fibre lasers, fibre sensors and optical coherence tomography systems. In this letter, we present an investigation of the high temperature dependence of an optical microfibre coupler with the objective determining if such a coupler could be used as an accurate temperature sensor for high temperature measurement from $700{ }^{\circ} \mathrm{C}$ to in excess of $1000^{\circ} \mathrm{C}$. It is well know that high temperature sensing circa $1000^{\circ} \mathrm{C}$ using optical fibre based sensors is challenging, many fibre sensors commonly used for lower temperature sensing cannot be used at such high temperatures, for example FBGs written in silica glass suffer grating erasure following exposure to such high temperatures.

In our experiment, low-loss microfibre couplers were manufactured from standard Corning SMF-28 singlemode fibres with the aid of the well-established single stage "flame-brushing" technique [3]. The longitudinal profile of the conical transition tapers was approximated by a decreasing/increasing exponential function and was achieved by reliable control of the hot zone and precise movement of the stages. The waist diameters of the fabricated microfibre couplers were circa $3 \mu \mathrm{m}, 2.5$ $\mu \mathrm{m}$ and $1.9 \mu \mathrm{m}$, respectively; the length of tapered uniform waist was $5 \mathrm{~mm}$.

Intially, prior to investigating the temperature characteristics of the microfibre couplers, it is useful to investigate modal interference in microfibre couplers. For this the output transmission spectra were measured for various diameters of the uniform waist region using a supercontinuum source (Fianium, wavelength coverage 450 1800 nm) in conjunction with an optical spectrum analyzer (YOKOGAWA AQ6370). Figure 1 shows the spectral outputs of a microfibre coupler made from a standard SMF28 singlemode fibre for different diameters of the uniform waist region: the significant modal interference induced by mode coupling between the lower-order symmetric and anti-symmetric supermodes can be observed when the diameter of the waist is decreased from 3 to $1.9 \mu \mathrm{m}$ : both the period and strength of the sinusoidal modulation in the transmission spectrum significantly decreased with a reduction in the waist diameter. 


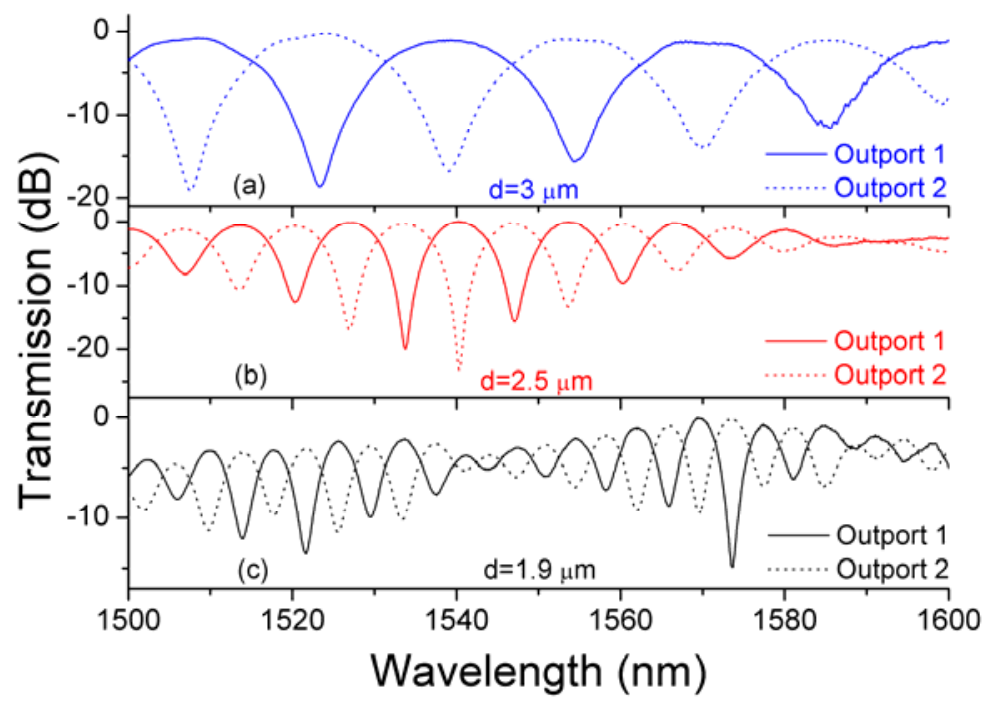

Fig. 1 (Color online) Spectral response of microfibre couplers: Transmission spectra of output ports for different microfibre diameters at (a) $3 \mu \mathrm{m}$, (b) $2.5 \mu \mathrm{m}$ and (c) $1.9 \mu \mathrm{m}$.

To examine its suitability as a sensor the high temperature $(\mathrm{T})$ characteristics of the microfibre coupler were tested by placing the uniform tapered region of the microfibre coupler in a resistive heater. Figure 2(a) shows the transmission spectra of the microfibre coupler at $\mathrm{T} \sim 701^{\circ} \mathrm{C}, 865^{\circ} \mathrm{C}$ and $1029^{\circ} \mathrm{C}$, respectively. For increasing T, the dip A redshifts from 1507.9 to $1516.5 \mathrm{~nm}$, while the background loss decreases, possibly because of changes induced by the thermal expansion of the silica and the thermo-optic effect. Figure 2(b) shows the experimental relationship between temperature and the resonant wavelength shift. The experimental temperature sensitivities of microfibre couplers with a diameter of $3 \mu \mathrm{m}, 2.5 \mu \mathrm{m}$ and $1.9 \mu \mathrm{m}$ are estimated as $25.91,36.59$ and $31.1 \mathrm{pm} /{ }^{\circ} \mathrm{C}$, respectively. The temperature sensitivity of the microfibre coupler is about 3.6 times higher than that of an $\mathrm{FBG}\left(\sim 10 \mathrm{pm} /{ }^{\circ} \mathrm{C}\right)[4]$, therefore this microfibre coupler temperature sensor could be also used for high temperature monitoring, without the risk of grating erasure associated with silica glass based FBGs 

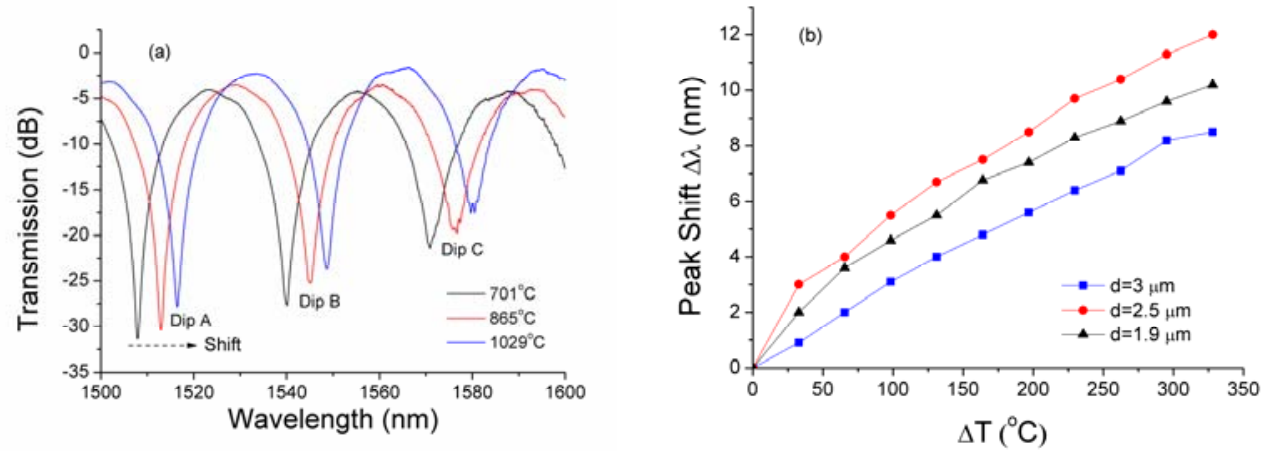

Fig. 2. (Color online) (a) Spectral responses of microfibre coupler with an outer diameter of $3 \mu \mathrm{m}$ (output port 1) vary with three different temperatures; (b) Measured peak wavelength shifts versus the temperature changes for microfibre couplers with different microfibre outer diameters.

In conclusion, the high temperature dependence of an optical microfibre coupler has been investigated experimentally. Used as the basis of a high temperature sensor, the temperature measurement sensitivity can reach up to $36.59 \mathrm{pm} /{ }^{\circ} \mathrm{C}$ for a taper region outer diameter of $2.5 \mu \mathrm{m}$.

\section{Acknowledgement}

Pengfei Wang is funded by the Irish Research Council for Science, Engineering and Technology, co-funded by the European Union Marie-Curie Actions under FP7. Q. Wu gratefully acknowledges the support of Science Foundation Ireland under Grant no. 07/SK/I1200.

\section{References}

1. G. Brambilla, "Optical fibre nanowires and microwires: a review", Journal of Optics, Vol. 12, No. 4, pp.043001, (2010).

2. Y. Jung, G. Brambilla, D. J. Richardson, "Optical microfiber coupler for broadband single-mode operation", Optics Express, Vol.17, No. 7, pp.5273-5278 (2009).

3. G. Brambilla, V. Finazzi, and D. J. Richardson, "Ultra-low-loss optical fiber nanotapers,” Opt. Express, Vol. 12, No. 10. pp. 2258-2263, (2004).

4. L. Men, P. Lu and Q. Chen, "A multiplexed fiber Bragg grating sensor for simultaneous salinity and temperature measurement," J. Appl. Phys., Vol. 103, pp. 053107, (2008). 
是在平台同向移动的过程中均匀拉伸的, 如果猛然拉伸的话, 会拉成竹节状的 taper, 另外猛然拉伸后taper里面形成的应力散射点会大大增多, 从而增大 taper 的传输损耗。平台移动的速度我之前跟你说过, 根据alpha值的不同, 移动速度 也不同, 你再仔细检查一下我给你的源代码, 看看里面是否有写? 如果alpha值 等于 0 的话, 我目测平台同向移动的速度应该是 $3 \sim 4 \mathrm{~mm} /$ 秒。

如果Ginu临走的时候搞不定, 等我回去的时候再说。 